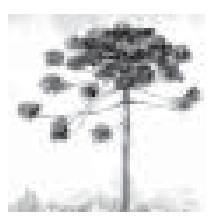

\title{
PANORAMA DA COLPOCITOLOGIA ONCÓTICA NA REDE PÚBLICA DO ESTADO DE SANTA CATARINA, BRASIL
}

\author{
A n overview of oncotic colpocytology in the State of \\ Santa Catarina public health, Brazil
}

\author{
Sabrina Gonçalves ${ }^{1}$, Romilton Jasper Rech ${ }^{2}$, Silvio Sica ${ }^{3}$, Júlio Cezar Merlin ${ }^{4}$, Patrícia Haas ${ }^{5}$ \\ ${ }^{1}$ Mestre em FarmáciapelaUFSC. Florianópolis, SC - Brasil. \\ ${ }^{2}$ Bioquímico do Laboratório Santa Maria- Turvo, SC - Brasil. \\ ${ }^{3}$ Bioquímico do Laboratório Bioclínico- Campo E rê, SC - Brasil. \\ ${ }^{4}$ Professor da Disciplina de Citologia Clínica da PUCPR. Curitiba, PR - Brasil. \\ ${ }^{5}$ Professora do D epartamento de Análises Clínicas (UFSC).Florianópolis, SC - Brasil, e-mail: haas@ ccs.ufsc.br
}

\begin{abstract}
Resumo
O exame colpocitológico adquiriu papel de destaque na prevenção do câncer ginecológico por possibilitar um diagnóstico precoce, condição essencial para o sucesso terapêutico. 0 objetivo deste estudo foi traçar um panorama dos resultados, observados na colpocitologia oncótica, na rede pública do E stado de Santa Catarina e avaliar a consolidação do Programa Viva Mulher. As informações utilizadas, referentes ao período de 2002 a 2006, foram obtidas a partir de consultas ao banco de dados do D epartamento de Informática do SUS - DATASUS, sendo considerados aspectos como citologia anterior, adequabilidade do esfregaço, números notificados de casos de suspeita de HPV e anormalidades de células epiteliais. Pode-se observar que, no período estudado, $71,7 \%$ das mulheres já haviam anteriormente se submetido ao exame. D os 1.791.634 exames realizados, aproximadamente 1.147.557 apresentaram-se satisfatórios para avaliação. As atipias em células escamosas de significado indeterminado e lesões escamosas intraepiteliais observadas, tiveram índices de 1,2\% e 0,8\%, respectivamente. Nas mulheres catarinenses houve alta prevalência de cervicites por $\mathrm{G}$ ardnerella vaginalis $(67,4 \%)$, e a faixa etária mais acometida pelos efeitos citopáticos do HPV foi entre 20 e 29 anos. A adequação da amostra constitui ponto essencial para um diagnóstico seguro. As atipias criam, tanto para o médico quanto para a paciente, um sentimento de incerteza e de insegurança. Tanto os achados de cervicites por $\mathrm{G}$ ardnerella vaginalis quanto a faixa etária mais acometida pelo HPV corroboram os dados propostos pela literatura. Os dados apresentados sugerem que ainda são crescentes as limitações de avaliação dos esfregaços e permitem inferir que a compilação de dados foi facilitada, embora ainda apresente algumas falhas que podem comprometer a confiabilidade dos resultados.
\end{abstract}

Palavras-chave: Colpocitologia oncótica; Epidemiologia; Cervicites. 


\begin{abstract}
The colpocytologic examinations acquired role of prominence in the prevention of the gynecological cancer for making possible a previous diagnosis, essential condition for the therapeutic success. The objective of this study was to trace a prospect of the results observed in the oncotic colpocytology in the State of Santa Catarina public health and to evaluate the consolidation of the Programa Viva Mulher. The information, referring to the period of 2002 at 2006, had been collected of the Departamento de Informática do SUS - DATASUS. Aspects as: previous cytology, suitableness of the smears, prevalence by age, notified numbers of HPV suspicion, abnormalitys in epithelial cells and vaginosis were considered. It may be observed that in the studied period, $71,7 \%$ of the women already had previously submitted to the examination. It was observed that 1.147.557 of 1.791 .634 colpocytologic examinations had been presented satisfactory for evaluation. The atypical squamous cells of undetermined significance and squamous intraepithelial lesions had indices of $1,2 \%$ and $0,8 \%$, respectively. There was high prevalence of vaginosis by Gardnerella vaginalis $(67,4 \%)$ in the studied women and age more attack for the cytopathic effect of the HPV was between 20 and 29 years old. The adequacy of the sample is essential for a guarantee disgnostic. The atypias create, as for the doctor as for the patient, an unreliability and uncertainty feeling. As the findings of vaginosis by Gardnerella vaginalis as the age more attack by HPV corroborates the data considered for the literature. The presented data suggest that the limitations of evaluation of the smears are still increasing and allow inferring that the compilation of data was facilitated, even still presents some imperfections that may compromise the trustworthiness of the results.
\end{abstract}

Keywords: Oncotic colpocytology; Epidemiology; Cervicitis.

\section{INTRODUÇÃO}

Desde sua idealização e implantação na rotina laboratorial, o exame colpocitológico, adquiriu papel de destaque na prevenção do câncer ginecológico. Considerado responsável pela redução de casos fatais, em decorrência do acesso facilitado e boa sensibilidade, possibilita um diagnóstico precoce, condição essencial para o sucesso terapêutico (1).

A tualmente, a epidemiologia do câncer de colo uterino está bem estabelecida em relação à existência de um agente carcinogênico. Tanto os carcinomas escamosos, quanto os adenocarcinomas são uma consequência rara e desenvolvida em longo prazo, a partir de uma infecção viral com subtipos do Papilomavírus Humano (HPV) (2). Walboomers et al. (1999) (3), demonstraram que a prevalência mundial do DNA de HPV em carcinomas cervicais é de 99,7\%.

O Ministério da Saúde do Brasil estima que aproximadamente 25\% da população brasileira sexualmente ativa, esteja contaminada pelo HPV, sendo que há pesquisas em outros países sinalizando que esse número pode chegar a 50\% entre os adolescentes e adultos jovens (4).
O câncer cervical apresenta alto potencial de prevenção e cura, mas no Brasil continua a ser um problema de saúde pública, com 19.260 novos casos estimados para o ano de $2006(5,6)$. Isso porque apenas $30 \%$ das mulheres submetem-se ao exame citopatológico pelo menos três vezes na vida, o que resulta em diagnóstico já na fase avançada da moléstia em $70 \%$ dos casos (6).

Em geral, a incidência desta neoplasia está em declínio na maioria dos países, que possuem um sistema adequado de rastreamento da doença, baseado, sobretudo, na citologia de Papanicolaou (7).

\section{OBJETIVOS}

Traçar um panorama dos resultados observados na colpocitologia oncótica, na rede pública do Estado de Santa Catarina, no período de 2002 a 2006 e avaliar a consolidação do Programa Viva Mulher, através do Sistema de Informações do Câncer da Mulher - SISCA M. 
MATERIAL E MÉTODO

\section{Material}

As informações utilizadas neste estudo, referentes ao período de 2002 a 2006, foram obtidas a partir de consultas ao banco de dados do Departamento de Informática do SUS - DATASUS. Através de um sistema informatizado de entrada de dados, denominado SistemadeInformaçõesdeCâncer da Mulher (SISCAM), aspectos como frequência das lesões pré-cancerosas, qualidade das coletas e das leituras das lâminas, puderam ser observadas (8).

O Sistema de Informação de Controle do Colo do Útero - SISCOLO, que é uma base de dados capaz de fornecer subsídios para a avaliação e planejamento do Programa Nacional de Controle do Câncer do Colo do Útero - Viva Mulher também foi utilizado nesse estudo.

\section{Nomenclatura utilizada}

Utilizou-seanomenclatura proposta pelo sistema Bethesda $(9,10)$ :

- ômalo é aquele que apresenta padrão citopatológico com atipias celulares de significado indeterminado (ASCUS), lesão intraepitelial de baixo grau (efeito citopático do HPV, displasia leve, NIC
I), lesão intraepitelial de alto grau (displasia moderada, displasia intensa, carcinoma in situ, NIC II, NIC III).

\section{Método}

Inicialmente, foram levantados dados de alterações citológicas e de flora vaginal a fim de se estabelecer um comparativo. Em seguida, alguns aspectos foram considerados como: citologia anterior, adequabilidade dos esfregaços, prevalência por faixa etária e números notificados de casos de alterações celulares sugestivas de infecção por HPV e de anormalidades em células epiteliais.

No Estado de Santa Catarina, além do número total de casos notificados de 2002 a 2006, a suspeita de infecção por HPV foi comparada em termos de prevalência a outros agentes infecciosos causadores de cervicites no mesmo período.

\section{RESULTADOS}

Conforme dados do SISCAM/ SISCOLO, pode-se observar que de 1.791.634 exames colpocitológicos realizados no Estado, no período citado, 897.991 mulheres foram investigadas quanto à realização de exame citopatológico anterior (Tabela 1).

TABELA 1 - Distribuição da população, segundo a realização do exame colpocitológico anterior no período de 2002 a 2006 e faixa etária, Santa Catarina, Brasil, 2007

\begin{tabular}{|c|c|c|c|c|c|c|}
\hline \multirow[t]{2}{*}{ Faixa etária } & \multicolumn{2}{|c|}{ Sim } & \multicolumn{2}{|c|}{ Não } & \multicolumn{2}{|c|}{ Não informaram } \\
\hline & $\mathbf{n}^{0}$ & $\%$ & $\mathbf{n}^{\circ}$ & $\%$ & $\mathbf{n}^{\circ}$ & $\%$ \\
\hline$<$ ou $=19$ anos & 33.412 & 2,7 & 38.375 & 3,1 & 10.246 & 0,8 \\
\hline 20 a 29 anos & 218.005 & 17,4 & 61.289 & 4,9 & 42.864 & 3,4 \\
\hline 30 a 39 anos & 252.787 & 20,2 & 34.733 & 2,8 & 44.630 & 3,6 \\
\hline 40 a 49 anos & 215.553 & 17,2 & 25.314 & 2,0 & 36.348 & 2,9 \\
\hline$>50$ anos & 178.234 & 14,2 & 24.077 & 1,9 & 34.736 & 2,8 \\
\hline Total & 897.991 & 71,7 & 183.788 & 14,7 & 168.824 & 13,5 \\
\hline
\end{tabular}

Fonte: Banco de dados do Departamento de Informática do SUS - DATASUS - SISCAM/ SISCOLO.

Quanto à adequabilidade das amostras, dos 1.791.634 exames citopatológicos realizados no Estado de Santa Catarina aproximadamente 1.147.557 apresentaram-se satisfatórios para avaliação porpossuírem $51 \%$ de células endocervicais ou 13\% de células metaplásicas e 644.077 (36\%) exames apresentaram-se insatisfatórios ou pela ausênciados grupos celulares anterioresou poroutros fatores como amostra não rotulada, obscurecida por sangue, entre outros (Figura 1). 


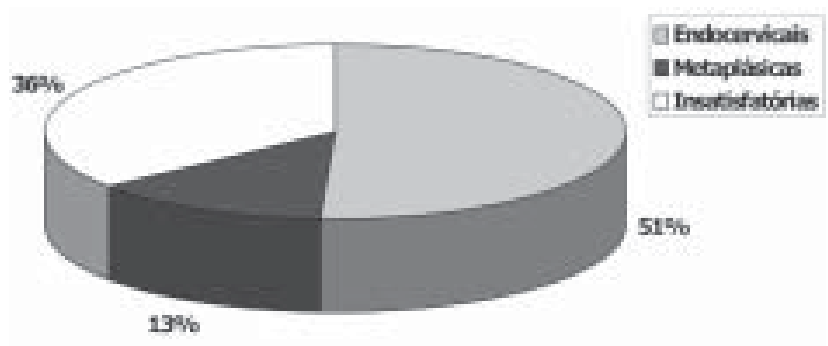

FIGURA 1 - Presença de células endocervicais ou metaplásicas nos esfregaços citopatológicos analisados no período de 2002 a 2006, Santa Catarina, Brasil, 2007

No total de 1.791.634 exames preventivos realizadosno Estado deSantaCatarinaidentificaramse: 22.200 ASCUS (células escamosas atípicas de significado indeterminado), 10.043 LSIL (lesão escamosa intraepitelial de baixo grau) e 4.700 HSIL (lesão escamosa intraepitelial de alto grau) em todo o período e também se verificou a prevalência dos mesmos por faixa etária (Figura 2).

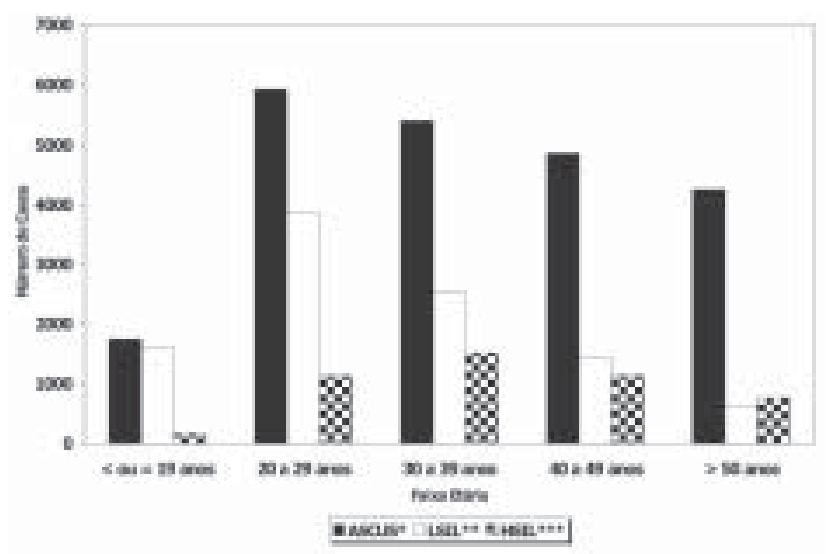

FIGURA 2 - Distribuição dos casos de anormalidades celulares segundo faixa etária no período de 2002 a 2006, Santa Catarina, Brasil, 2007

* Células Escamosas Atípicas de Significado Indeterminado

** Lesão Escamosa Intra-epitelial de Baixo Grau

*** Lesão Escamosa Intra-Epitelial de Alto Grau

Fonte: Banco de dados do D epartamento de Informática do SUS - DATASUS - SISCAM/ SISCOLO.

Nas mulheres catarinenses se verificou maior prevalência de cervicites por $\mathrm{G}$ ardnerella vaginalis $(67,4 \%)$, seguida de $C$ andida spp $(24,9 \%)$, Trichomonas vaginalis $(5,8 \%)$ e $\mathrm{HPV}(1,9 \%)$ no perío do avaliado (Figura 3).

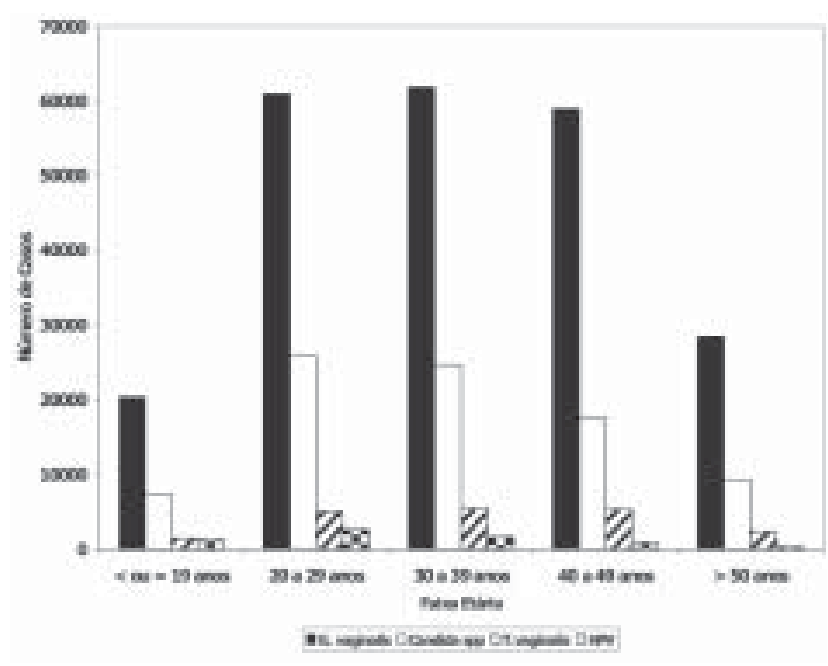

FIG URA 3 - D istribuição dos casos de cervicites segundo agente etiológico e faixa etária no período de 2002 a 2006, Santa Catarina, Brasil, 2007.

Fonte: Banco de dados do Departamento de Informática do SUS DATASUS - SISCAM/ SISCO LO.

A Figura 4 mostra a distribuição dos casos notificados de alterações celulares sugestivas de infecção por HPV nos grupos etários (Figura 4).

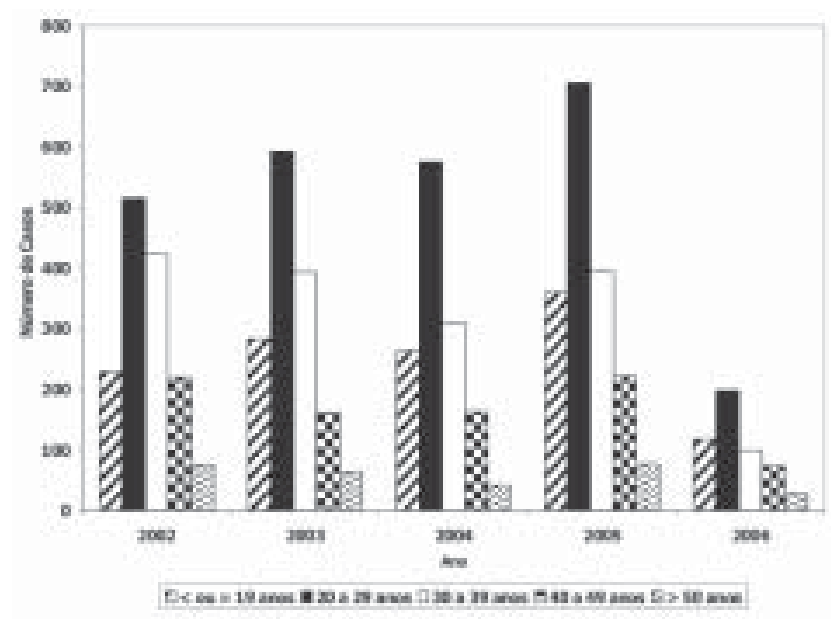

FIGURA 4 - D istribuição dos casos de Papilomavírus Humano (HPV) segundo ano e faixa etária no período de 2002 a 2006, Santa Catarina, Brasil, 2007

Fonte: Banco de dados do Departamento de Informática do SUS - DATASUS - SISCAM/ SISCOLO. 


\section{DISCUSSÃO}

Para se garantir a eficácia de um programa de prevenção populacional é necessário e essencial um alto nível de participação da população e pelas informações que coletamos isto foi possível. 0 controle do câncer cervical depende do número de mulheres que nunca realizaram o exame preventivo equeo programa podeatingir. Pelo queobservamos, no período estudado, o programa Viva Mulher precisava atingir ainda $28,2 \%$ das mulheres catarinenses, caso considerássemos as mulheres que não responderam como prováveis mulheres que não realizaram 0 exame anteriormente.

A adequação da amostra constitui ponto essencial paraum diagnóstico seguro. Vários aspectos estão envolvidos no que se refere a um esfregaço satisfatório para avaliação, cuja descrição é detalhada de forma própria e objetiva no sistema de Bethesda. Além da identificação apropriada das lâminas e informações clínicas relevantes, a composição celular do esfregaço é considerada primordial na avaliação de sua representatividade (1).

Como na junção escamo-colunar (JEC) origina-se a maioria das lesões precursoras do câncer de colo, a presença de um componente escamoso e endocervical garantiria uma amostra adequada dessa região, aumentando a probabilidade da detecção de anormalidades cervicais. Ainda, existem observações que indicam que espécime sem tais componentes também permite a identificação de atipias celulares (1).

O Sistema Bethesda estabelece, como um componente endocervical/ zona de transformação adequado, um mínimo de dois grupos celulares glandulares endocervicais e/ ou metaplásicas bem preservadas, sendo cada grupo composto por, pelo menos, 5 células, não se aplicando a esfregaços marcadamente atróficos, onde células metaplásicas e endocervicais nem sempre podem ser distintas de células parabasais $(9,10)$.

A presença de células escamosas atípicas de significado indeterminado é um diagnóstico de exclusão para o citopatologista, uma vez que os achadosnão são suficientementeclarosparapermitir um diagnóstico específico. Apesar do esforço para garantir critérios específicos na constituição do ASCUS, o uso desse termo pode diferir para cada patologista. No entanto, os diagnósticos de ASCUS não devem exceder duas a três vezes os índices de lesões intraepiteliais escamosas (SIL), ou seja, entre $2 \%$ e $3 \%(9,10)$.

As Atipias em células escamosas de significado indeterminado e SIL, observadas no Estado de Santa Catarina, tiveram índices de 1,2\% e 0,8\%, respectivamente, corroborando os índices propostos pela literatura $(9,10)$.

Em muitas amostras demonstrando ASCUS, as pacientes não apresentam lesões expressivas e as biópsias colposcópicas mostram-se normais, entretanto em uma proporção significante de pacientes, a anormalidade persistirá e em novas avaliações será possível detectar lesões precursoras de câncer $(9,10)$. Rossetti et al. (2000) (11), constataram na reexaminação de resultados citológicos, onde houve diagnóstico de ASCUS, um aumento napositividade paralesões pré-neoplásicas. Esses resultados indicam que a presença de ASCUS deve ser avaliada colposcopicamente e histologicamente, quando indicado (11).

O diagnóstico de ASCUS nas mulheres catarinenses apresentou maior prevalência acima de 40 anos, sendo que na faixa etária de 12 a 19 anos mostrou um discreto predomínio em relação ao diagnóstico de LSIL. Em estudos realizados por O eberst e G eisinger (1996) (12), foram encontradas altas porcentagens de ASCUS em adolescentes, sendo que McD aniel e colaboradores (1996) (13) sugerem cuidado especial no diagnóstico citológico de ASCUS nas pós-menopausadas.

Uma maior prevalência de ASCUS também foi observada na faixa etária de 20 a 39 anos, entretanto não há evidências na literatura que comprovem a maior predominância do diagnóstico de ASCUS, em relação ao diagnóstico de LSIL, nesse grupo etário; tal resultado se deve, provavelmente, à incapacidade do citologista de precisar a natureza das anomalias celulares por carência técnica do esfregaço ou devido à presença de alterações nucleares acentuadas de origem inflamatória, que particularmente ocorrem na tricomoníase e herpes tipo 2 ou ainda em decorrência das alterações pós-radiação, pósquimioterapia e pós-cirurgia que o esfregaço das amostras coletadas dessas mulheres poderiam, consequentemente apresentar (14). 
O s esfregaços corados pelo Papanicolaou são usados como screening e permitem uma sensibilidade de $90 \%$ e especificidade de $97 \%$ nas amostras vaginais e não cervicais, para diagnóstico da vaginose bacteriana (15). Vários processos infecciosos vaginais decorrem do desequilíbrio da microflora, tal como ocorre durante a gravidez. A infecção mais comum é causada por $\mathrm{G}$ ardnerella vaginalis cuja frequência varia entre mulheres não grávidas de 2 a $58 \%$ (16).

Em Santa Catarina, as mulheres com idade entre 20 e 49 anos foram as mais acometidas pela cervicite causada por $\mathrm{G}$ ardnerella vaginalis $(17,18)$. Segundo Limae colaboradores (2003) (19), a vaginose bacteriana acomete mulheres em idade reprodutiva, indicando possível influência dos hormônios sexuais na sua patogênese. Em contrapartida, Candioto et al. (2003) (20), observaram que essa bactéria é comumente encontrada em mulheres jovens, de $20 \mathrm{a}$ 24 anos e com vida sexual ativa. Podendo também ser encontrada em adolescentes virgens, comprovando que a transmissão não é somente sexual. Há relatos de que esse microrganismo pode fazer parte da flora vaginal normal, sendo encontrado em 40 a 50\% das mulheres clinicamente saudáveis (20).

A detecção de $\mathrm{G}$ ardnerella vaginalis nos esfregaços citológicos pode ser realizada através do encontro de células chamadas dueœlls, cujaprevalência pode estar em torno de 73\% dos casos (16).

Em nosso estudo a segunda causa mais comum de infecção genital foi a candidíase. A C andida spp é responsável por 20 a $25 \%$ dos corrimentos genitais, contudo, cerca de $75 \%$ das mulheres têm a infecção pelo menos uma vez na vida $(21,22)$.

Nossos achados em relação à tricomoníase corroboram os dados da literatura, em que sua maior incidência ocorre na faixa etária de 30 a 50 anos $(18,23,24)$.

A citologia é o método mais conveniente, rápido, econômico e sensível para a detecção das alterações celulares sugestivas de infecção por HPV no trato genital, contudo, o diagnóstico dos efeitos citopáticos do papilomavírus, em demasia, pode resultar em problema adicional. A principal causa seria interpretar qualquer halo citoplasmático como sendo coilocitose (alteração celular sugestiva de HPV), sem ser acompanhado de atipia nuclear $(9,15,25)$.

A distribuição dos casos registrados no SISCAM de alterações celulares sugestivas de infecção por HPV, observadas no exame preventivo, em Santa Catarina, por ano, foi a seguinte: em 2002, 22,2\% (1.463/ 6.584); em 2003, $22,7 \%$ (1.493/6.584); em 2004, 20,6\% (1.354/ 6.584); em 2005, 26,7\% (1.759/6.584) e em 2006, $7,8 \%(515 / 6.584)(17,25)$. Pode-se observar que a menor notificação de casos de suspeita de HPV ocorreu no ano de 2006, devido a, provavelmente, dois motivos: descuido no diagnóstico de infecção pelo vírus ou falta de adaptação dos municípios em notificar e compilar seus dados.

Lancelotti et al. (2000) (26), estimaram que as lesões por HPV representam $60 \%$ das atipias cervicais triadas nos esfregaços sistemáticos, porém, cerca de um terço dessas infecções podem passar despercebidas citologicamente, se o diagnóstico for baseado apenas no encontro de alterações coilocitóticas.

No período estudado, a faixa etária mais acometida pela papilomavirose foi de 20 a 29 anos em todos os anos analisados, a segunda de 30 a 39 anos e a terceira de 12 a 19 anos. Acima de 50 anos foi a faixa etária que apresentou menores índices de detecção do vírus $(17,25,27)$.

A infecção por HPV é maior entre adolescentes e mulheres jovens devido ao precoce início da atividade sexual e ao número de parceiros $(24,28,29)$, porém, essa infecção também pode ocorrer em idades mais avançadas (28). A hipótese apoiadaporalguns pesquisadores éde que o primeiro coito em idade precocepoderia significarum epitélio cervical puberal mais suscetível à agressão oncogênica (29).

Em alguns estudos observou-se que a prevalência de HPV entre todas as mulheres e em mulheres acima dos 30 anos foi de 15,1\% e 9,2\%, respectivamente. Nos Estados Unidos e no oeste da Europa a prevalência do vírus declina agudamente e atinge níveis muito baixos, próximo dos 50 anos, conforme a imunidade da paciente $(28,29,30)$.

\section{CONCLUSÕES}

A análise dos aspectos observados na população estudadasugeriu queainda são crescentes as limitações de avaliação dos esfregaços por haver em alguns casos coleta inadequada, má fixação, erro de leitura pelos citopatologistas ou citotécnicos inexperientes ou ainda devido a condições desfavoráveis da paciente, como infecção e traumatismo, que podem levar a uma conclusão equivocada (1). 
Além disso, a classificação do esfregaço na categoria "atípica" cria, tanto para o médico quanto para a paciente, um sentimento de incerteza e de insegurança enão permitedefinirumacondutaclínica adequada (14). Portanto, deve-se evitar, sempre que possível, lançar mão desse diagnóstico, pois o exame citológico é o método mais acessível para a maioria da população brasileira que utiliza a rede pública.

De modo geral as informações coletadas propiciam fazerapenas um panoramadosresultados dos exames colpocitológicos realizados na rede pública, pois eventuais erros de registro dos dados podem ocorrer, como falta de atenção na transferência das informações contidas nos laudos dos exames citopatológicos para 0 aplicativo SISCAM, bem como, também se deve levar em consideração queamesmamulher podeter realizado mais de um exame no período.

Entretanto, o SISCAM facilitou a compilação de dados, embora ainda apresente algumas falhas que podem comprometer a confiabilidade dos resultados, mas o Programa Viva Mulher, juntamente com o SISCO LO, vem buscando ações estratégicas para garantir maior abrangência de certificações dos casos e com isso consolidar o programa, quealém de obter os dados poderá aferi-los quantitativa e qualitativamente.

Como o Programa Viva Mulher consiste na formação de profissionais de saúde capacitados para estimular a prevenção, realizar a detecção precoce e o tratamento do câncer de colo uterino, os dadospornós levantados indicaram umaboaadesão a esta atenção preventiva, por parte das mulheres catarinenses, contudo, deve-se dar maior ênfase e expansão às campanhas preventivas.

\section{REFERÊNCIAS}

1. Silva HA, Silveira LMS, Pinheiro VMF, Mendes APS, Ribeiro WR, et al. Papilomavírus humano e lesões intra-epiteliais cervicais: estudo colpocitológico retrospectivo. RBAC. 2003;35(3):117-21.

2. Rabelo S. HPV: uma estatística preocupante. J SBAC. 2002.12:2.

3. Walboomers JM, Jacobs MV, Manos MM, Bosch FX, Kummer JA, Shah KV, Snijders $\mathrm{PJ}$, et al. Human papillomavirus is a necessary cause of invasive cervical cancer worldwide. J Pathol. 1999;189(1):1-3.
4. Aguiar R. Controle da infecção por HPV pode prevenir câncer de colo de útero. [Internet]. Site da Fiocruz. [citado 20 de março de 2005]. Disponível em: http:/ / www.fiocruz.br/ ccs/ novidades/ dez03/ hpv.htm

5. Instituto Nacional do Câncer - INCA. Câncer de colo de útero. [Internet]. Site do INCA. [citado 10 de setembro de 2007]. D isponível em: http:/ / www.inca.gov.br

6. Motti E. et al. Câncer de colo do útero. Boletim Informativo Brasil [Internet]. 2003 [2006 fev. 22];5:4. Disponível em: http:/ / www.eurotrials.com/ publicacoes/ bolbrpt5.pdf

7. Centro de Tratamento e Pesquisa Hospital do Câncer - AC Camargo. Site do INCA [Internet]. [citado 2005 jan 20]. Disponível em: http:/ / hcanc.org.br

8. Ministério da Saúde. Sistema de informações de câncer da mulher (SISCAM/ SISCO LO) [Internet]. Site do Ministério da Saúde [citado ago. 27]. Disponível em: http:// www.datasus.gov.br

9. Kurman RJ, Solomon D O. Sistema Bethesda para o relato de diagnóstico citológico cérvicovaginal. Rio de Janeiro: Revinter; 1997.

10. Solomon D, Nayar R. Sistema Bethesda para citopatologia cervicovaginal. $2^{\text {a }}$ ed. Rio de Janeiro. Revinter; 2005.

11. Rossetti D, et al. Atypical squamous cells of undetermined significance (ASCUS), low-grade squamous intraepithelial lesion (LSIL), high-grade squamous intraepithelial lesion (HSIL) and histology. J Med Liban. 2000; 48(3):127-30.

12. O eberst JL, G eisinger KR. Histologic and cytologic follow up in teenagers with atypical squamous cells of undetermined significance (ASCUS) on cervical smears. Acta Cytol. 1996;40:1032.

13. Mcdaniel ML, et al. Atypical squamous cells of undetermined significance (ASCUS) and postmenopausal PAP smear: separation of squamous intraepithelial lesion (SIL) from atrophic changes. Acta Cytol. 1996; 40:1021.

14.Gompel C, Koss LG. Citologia ginecológica e suas bases anatomoclínicas. São Paulo: Manole; 1997. p. 101-2. 
15. Silva Filho AM, Longatto Filho A. Colo uterino e vagina. Processos inflamatórios. Rio de Janeiro: Revinter; 2000.

16. Murta EFC, et al. Incidence of $\mathrm{G}$ ardnerella vaginalis, $C$ andida sp and human papilloma virus in cytological smears. São Paulo Med J. 2000;118(4):105-8.

17. Silveira, MD P. Panorama dos resultados de casos de HPV notificados na rede pública do Estado de Santa Catarina. In: $31^{\circ}$ Congresso Brasileiro de Análises Clínicas e $4^{\circ}$ Congresso Brasileiro de Citologia Clínica. Salvador BA, 2004. p. 54.

18. Gonçalves S, Haas P, Perfil E pidemiológico dos Casos de Cervicites por $\mathrm{C}$ andida spp, $\mathrm{G}$ ardnerella vaginalis e Trichomonas vaginalis notificados pelo Sistema Público de Saúde na Região Sul- Brasil. In: $34^{\circ}$ Congresso Brasileiro de Análises Clínicas. Belo Horizonte - MG, 2007. p. 22.

19.Lima GR. Ginecologia de consultório. São Paulo: Projetos Médicos; 2003.

20. Candioto CAF. Infecção genital por $G$ ardnerella vaginalis. Rev FMT. 2003;5(1):14-9.

21.Boatto HF. Correlação entre os resultados laboratoriais e os sinais e sintomas clínicos das pacientes com candidíase vulvovaginal e relevância dos parceiros sexuais na manutenção da infecção em São Paulo, Brasil. RBGO. 2007;29(2):80-4.

22.Rosa MI, Rumel D. Fatores associados à candidíase vulvovaginal: estudo exploratório. RBG 0. 2004;26(1):65-70.

23. Franklin TL, Monif GG. Trichomonas vaginalis and bacterial vaginosis. Coexistence in vaginal wet mount preparations from pregnant women. The Journal of Reprodutive Medicine. 2000;45(2):131-4.

24. Stinghen AEM, Nascimento AJ, Leonart MSS. Método de papanicolaou em material cérvicovaginal para a triagem de infecção por $C$ andida sp., T richomonas vaginalis e $\mathrm{Chlamydia}$ trachomatis. RBAC. 2004;36(2):111-5.
25. Hastreiter AA. Perfil epidemiológico dos casos de cervicites e HPV notificados no sistema público de saúde do Estado de Santa Catarina. In: $31^{\circ}$ Congresso Brasileiro de Análises Clínicas. Salvador - BA, 2004. p. 55.

26.Lancellotti CLP. D ados epidemiológicos. In: I Consenso Brasileiro de HPV. São Paulo: Bg Cultural; 2000. p. 45-60.

27. Hastreiter AA. Avaliação da prevalência dos casos de HPV e neoplasias intra-epiteliais cervicais notificados nos Estados da Região Sul. In: $1^{0}$ Congresso Sul-Sudeste de Análises Clínicas. Campos do Jordão; 2005. p. 34.

28. Baseman JG, Koutsky LA. The epidemiology of human papillomavirus infections. J Clin Virology. 2005;32(Suppl 1):S16-24.

29. Schiffman M, Castle PE. Human papillomavirus. Epidemiology and public health. Arch Pathol Lab Med. 2003;127(8):930-4.

30.Zeferino LC. Epidemiologia da neoplasia intra-epitelial cervical. Rev Ginecol Obst. 1990;1(1):22-32.
Recebido: 07/ 01/ 2007

Received: 01/ 07/ 2007

Aceito: 25/ 02/ 2007

A ccepted: 02/ 25/ 2007 